\title{
Bayesian parameter estimation of Euler-Bernoulli beams
}

\author{
Iman T. Ardekani ${ }^{1}$, Jari Kaipio² ${ }^{2}$ Neda Sakhaee ${ }^{2}$, and Hamid Sharifzadeh ${ }^{1}$ \\ ${ }^{1}$ Unitec Institute of Technology, New Zealand \\ ${ }^{2}$ The University of Auckland, New Zealand
}

\begin{abstract}
This paper develops a statistical signal processing algorithm for parameter estimation of Euler-Bernoulli beams from limited and noisy measurement. The original problem is split into two reduced-order sub-problems coupled by a linear equation. The first sub-problem is cast as an inverse problem and solved by using Bayesian approximation error analysis. The second sub-problem is cast as a forward problem and solved by using the finite element technique. An optimal solution to the original problem is then obtained by coupling the solutions to the two sub-problems. Finally, a statistical signal processing algorithm for adaptive estimation of the optimal solution is developed. Computer simulation shows the effectiveness of the proposed algorithm.
\end{abstract}

Keywords: system identification, Bayesian approximation error, statistical signal processing, Euler-Bernoulli beams

\section{INTRODUCTION}

In many engineering applications, it is desired to identify external forces (force reconstruction problem) or mechanical properties (parameter estimation problem) of a structural element from limited and noisy measurement of deflection. In practice, deflection signal can be measured by using traditional strain gauges or advanced imaging techniques such as Photogrammetry. Usually, both the force reconstruction and parameter estimation problems lead to ill-posed mathematical problems; which should be treated by using regularization techniques such as Truncated Singular Value Decomposition (TSVD) ${ }^{1}$ or Tikhonov regularization. ${ }^{2}$ These techniques require intensive computations. Moreover, they require some regularization parameters to be adjusted by using some prior knowledge of solution. There exist a reach literature on the force reconstruction in various structural elements including beams and plates; ${ }^{3}$ however, the literature on the parameter estimation is rather limited. This is mainly because the force reconstruction problem can be cast as a linear de-convolution problem ${ }^{4}$ but the parameter estimation problem is usually cast as a nonlinear inverse problem. ${ }^{5}$

Particular solutions for the parameters estimation of Euler-Bernoulli beams can be found in papers published by Lesnic ${ }^{6,7}$ or Marinov. ${ }^{8,9}$ They developed novel mathematical models based on which system parameters can be derived through a regularized optimization process. Unfortunately, these solutions cannot be realized in forms of efficient signal processing algorithms because of their intensive computation. Furthermore, their performance is highly depended on the regularization parameters. These parameters are very sensitive to the input data so they should be adjusted for every set of new measurement data at each iteration. This process requires intensive computation and prior knowledge of solution. Finally, standard regularization techniques are unable to consider modeling error or measurement noise.

The focus of this paper is on developing an efficient signal processing algorithm for parameter estimation of Euler-Bernoulli beams considering modeling error and measurement noise. Section 2 creates a novel model for the parameter estimation of Euler-Bernoulli beams by splitting the original problem into two coupled reducedorder sub-problems. Section 3 gives a novel solution to the first sub-problem based on Bayesian analysis of approximation error. ${ }^{10}$ Section 4 gives a solution to the second sub-problem and develops an efficient signal processing algorithm for online parameter estimation of Euler-Bernoulli beams. Section 5 discusses computer simulations. Finally, Section 6 gives concluding remarks. 


\section{MATHEMATICAL MODELING}

Referring to Fig. 1a, a cantilever Euler-Bernoulli beam of length 1 is modeled by the boundary value problem of

$$
\left\{\begin{array}{l}
\left(\beta u^{\prime \prime}\right)^{\prime \prime}=f, \quad x \in[0,1] \\
u(0)=u^{\prime}(0)=u^{\prime \prime}(1)=u^{\prime \prime \prime}(1)=0
\end{array}\right.
$$

where $f(x), u(x)$ and $\beta(x)$ are the force, deflection and flexural rigidity functions. The parameter estimation problem associated with Eq. (1) is to find $\beta(x)$ given $u(x)$ and $f(x)$. In this paper, it is proposed to express the 4th-order model given in Eq. (1) as two 2nd-order sub-models,

$$
\begin{gathered}
\mathcal{M}_{1}:\left\{\begin{array}{l}
u^{\prime \prime}(x)=g(x) \\
u(0)=u^{\prime}(0)=0
\end{array}\right. \\
\mathcal{M}_{2}:\left\{\begin{array}{l}
m^{\prime \prime}(x)=f(x) \\
m(1)=m^{\prime}(1)=0
\end{array}\right.
\end{gathered}
$$

that are coupled by

$$
m(x)=\beta(x) g(x)
$$

The problem associated with the first sub-model (first sub-problem) is cast as an inverse problem to find $g(x)$ given a limited and noisy measurement of $u(x)$. The problem associated with the first sub-model (second subproblem) is cast as a forward problem to find $m(x)$ given $f(x)$. Once $g(x)$ and $m(x)$ are found, the parameter function $\beta(x)$ can be then reconstructed from the coupling equation given in Eq. (4).

\section{BAYESIAN APPROXIMATION ERROR ANALYSIS}

Let us assume that beam deflection is measured at $x_{1}, \ldots, x_{M}$ and a measurement vector $\mathbf{r} \in \mathbb{R}^{M}$ is formed by using the measurement data. The measurement vector can be modeled by

$$
\mathbf{r}=\mathbf{u}+\mathbf{e}
$$

where $\mathbf{u}=\left[u\left(x_{1}\right), \ldots, u\left(x_{M}\right)\right]^{T}$ is a rough model for the measurement data and e models all the uncertainty associated with the process (or "approximation error") including modeling error and measurement noise. A rough model for $u(x)$ can be given by

$$
u(x)=\sum_{k=1}^{N} \mathrm{a}_{k} \varphi_{k}(x)
$$

where $\varphi_{1}, \ldots, \varphi_{N}$ are mode functions (or "basis functions") and $\mathrm{a}_{1}, \ldots, \mathrm{a}_{N}$ are scalar coefficients. From Eqs. (6), $\mathbf{u}=\Psi \mathbf{a}$ where $\Psi=\left[\varphi_{j}\left(x_{i}\right)\right]_{M \times N}$; thus, Eq. (5) leads to

$$
\mathbf{r}=\Psi \mathbf{a}+\mathbf{e}
$$

Substituting Eq. (6) into Eq. (2) and using Galerkin method results in

$$
\mathbf{P a}=\mathbf{g} \Rightarrow \mathbf{a}=\mathbf{P}^{-1} \mathbf{g}
$$

where $\mathbf{P}=\left[\mathrm{P}_{k l}\right]_{N \times N}$ and $\mathbf{g}=\left[\mathrm{g}_{l}\right]_{N \times 1}$ are given by $\mathrm{P}_{k l}=\varphi_{k}^{\prime}(1) \varphi_{l}(1)-\int_{0}^{1} \varphi_{k}^{\prime}(x) \varphi_{l}^{\prime}(x) \mathrm{d} x$ and $\mathrm{g}_{l}=\int_{0}^{1} g(x) \varphi_{l}(x) \mathrm{d} x$. From Eqs. (7) and (8), the discrete form of the first sub-problem is obtained by

$$
\mathbf{r}=\mathbf{K g}+\mathbf{e}
$$

where $\mathbf{K}=\Psi \mathbf{P}^{-1}$ is a matrix in $\mathbb{R}^{M \times N}$. This problem can be cast as to find $\mathbf{g}$ (modal values of $g$ ) given $\mathbf{r}=\mathbf{u}+\mathbf{e}$ (noisy measurement of $u$ at limited number of points), where $\mathbf{e}$ is an stochastic variable. Standard regularization 
techniques such as Tikhonov regularization are unable to consider e in Eq. (9). To overcome this weakness, a novel method based on Bayesian analysis of approximation error is proposed in the following. From Bayes' rule a posterior density for $\mathbf{g}$ given the measurement vector $\mathbf{r}$ is

$$
\pi(\mathbf{g} \mid \mathbf{r}) \propto \pi(\mathbf{r} \mid \mathbf{g}) \pi(\mathbf{g})
$$

Eq. (9) results in $\pi(\mathbf{r} \mid \mathbf{e}, \mathbf{g})=\delta(\mathbf{r}-\mathbf{K g}-\mathbf{e})$. Substituting this result into $\pi(\mathbf{r}, \mathbf{e} \mid \mathbf{g})=\pi(\mathbf{r} \mid \mathbf{e}, \mathbf{g}) \pi(\mathbf{e} \mid \mathbf{g})$ gives

$$
\pi(\mathbf{r}, \mathbf{e} \mid \mathbf{g})=\delta(\mathbf{r}-\mathbf{K g}-\mathbf{e}) \pi(\mathbf{e} \mid \mathbf{g})
$$

Integration over e from the both sides of Eq. (11) results in

$$
\int_{\mathbb{R}^{M}} \pi(\mathbf{r}, \mathbf{e} \mid \mathbf{g}) \mathrm{d} \mathbf{e}=\int_{\mathbb{R}^{M}} \delta(\mathbf{r}-\mathbf{K g}-\mathbf{e}) \pi(\mathbf{e} \mid \mathbf{g}) \mathrm{d} \mathbf{e}
$$

In Eq. (12), the LHS is equal to $\pi(\mathbf{r} \mid \mathbf{g})$ and the RHS can be simplified by using the properties of the delta function; thus,

$$
\pi(\mathbf{r} \mid \mathbf{g})=\pi_{\mathrm{e} \mid \mathrm{g}}(\mathbf{r}-\mathbf{K g}-\mathbf{e})
$$

where the notation $\pi_{\mathrm{e} \mid \mathrm{g}}(\mathbf{x})$ is used to show $\left.\pi(\mathbf{e} \mid \mathbf{g})\right|_{\mathbf{e}=\mathbf{x}}$. By definition,

$$
\pi(\mathbf{e} \mid \mathbf{g})=\frac{\pi(\mathbf{e}, \mathbf{g})}{\pi(\mathbf{g})}
$$

Let us assume $\mathbf{g}$ and $\mathbf{e}$ follow Gaussian densities: $\pi(\mathbf{g})=\mathcal{N}\left(\mathbf{g}_{*}, \Gamma_{\mathrm{gg}}\right), \pi(\mathbf{e})=\mathcal{N}\left(\mathbf{e}_{*}, \Gamma_{\mathrm{ee}}\right)$. In this case the joint density $\pi(\mathbf{e}, \mathbf{g})$ is in the form of

$$
\pi(\mathbf{e}, \mathbf{g}) \propto \exp \left(-\frac{1}{2}\left[\begin{array}{c}
\mathbf{e}-\mathbf{e}_{*} \\
\mathbf{g}-\mathbf{g}_{*}
\end{array}\right]^{T}\left[\begin{array}{ll}
\Gamma_{\mathrm{ee}} & \Gamma_{\mathrm{eg}} \\
\Gamma_{\mathrm{ge}} & \Gamma_{\mathrm{gg}}
\end{array}\right]^{-1}\left[\begin{array}{c}
\mathbf{e}-\mathbf{e}_{*} \\
\mathbf{g}-\mathbf{g}_{*}
\end{array}\right]\right)
$$

where $\Gamma_{\text {eg }}$ is the co-variance matrix of $\mathbf{g}$ and $\mathbf{e}$. All the statistical parameters used in Eq. (15) can be computed numerically. This process is discussed in detail in Section 4. Eq. (15) can be re-arranged to

$$
\pi(\mathbf{e}, \mathbf{g}) \propto \exp \left\{-\frac{1}{2}\left(\left(\mathbf{e}-\mathbf{e}_{* \mid \mathrm{p}}\right)^{T} \Gamma_{\mathrm{r} \mid \mathrm{p}}^{-1}\left(\mathbf{e}-\mathbf{e}_{* \mid \mathrm{p}}\right)+\left(\mathbf{g}-\mathbf{g}_{*}\right)^{T} \Gamma_{\mathrm{gg}}^{-1}\left(\mathbf{g}-\mathbf{g}_{*}\right)\right)\right\}
$$

where conditional mean $\mathbf{e}_{* \mid \mathrm{g}}$ and conditional co-variance $\Gamma_{\mathrm{e} \mid \mathrm{g}}$ are given by

$$
\left\{\begin{array}{l}
\mathbf{e}_{* \mid \mathrm{g}}=\mathbf{e}_{*}+\Gamma_{\mathrm{eg}} \Gamma_{\mathrm{gg}}^{-1}\left(\mathbf{g}-\mathbf{g}_{*}\right) \\
\Gamma_{\mathrm{e} \mid \mathrm{g}}=\Gamma_{\mathrm{ee}}-\Gamma_{\mathrm{eg}} \Gamma_{\mathrm{gg}}^{-1} \Gamma_{\mathrm{ge}}
\end{array}\right.
$$

Now, combining Eqs. (13), (14) and (16) results in

$$
\pi(\mathbf{r} \mid \mathbf{g}) \propto \exp \left(-\frac{1}{2}\left(\mathbf{r}-\mathbf{K g}-\mathbf{e}_{* \mid \mathrm{g}}\right)^{T} \Gamma_{\mathrm{e} \mid \mathrm{g}}^{-1}\left(\mathbf{r}-\mathbf{K g}-\mathbf{e}_{* \mid \mathrm{g}}\right)\right)
$$

Substituting Eq. (18) into (10) and using $\pi(\mathbf{g})=\mathcal{N}\left(\mathbf{g}_{*}, \Gamma_{\mathrm{gg}}\right)$ results in

$$
\pi(\mathbf{g} \mid \mathbf{r}) \propto \exp \left(-\frac{1}{2}\left(\mathbf{r}-\mathbf{K g}-\mathbf{e}_{* \mid \mathrm{g}}\right)^{T} \Gamma_{\mathrm{e} \mid \mathrm{g}}^{-1}\left(\mathbf{r}-\mathbf{K g}-\mathbf{e}_{* \mid \mathrm{g}}\right)-\frac{1}{2}\left(\mathbf{g}-\mathbf{g}_{*}\right)^{T} \Gamma_{\mathrm{gg}}^{-1}\left(\mathbf{g}-\mathbf{g}_{*}\right)\right)
$$

A Maximum A Posteriori (MAP) estimation of $\mathbf{g}$, denoted by $\mathbf{g}^{M A P}$, can be obtained by maximizing $\pi(\mathbf{g} \mid \mathbf{r})$. Note that $\mathbf{e}_{* \mid \mathrm{g}}$ is a function of $\mathbf{g}$ as shown in Eq. (17). After maximizing (19), $\mathbf{g}^{M A P}$ is obtained by

$$
\mathbf{g}^{M A P}=\boldsymbol{\Lambda}^{-1}\left[\boldsymbol{\kappa}\left(\mathbf{r}-\mathbf{e}_{*}\right)+\lambda \mathbf{g}_{*}\right]
$$

where

$$
\left\{\begin{array}{l}
\boldsymbol{\kappa}=\left(\mathbf{K}+\Gamma_{\mathrm{eg}} \Gamma_{\mathrm{gg}}^{-1}\right)^{T} \Gamma_{\mathrm{e} \mid \mathrm{g}}^{-1} \\
\boldsymbol{\Lambda}=\boldsymbol{\kappa} \Gamma_{\mathrm{e} \mid \mathrm{g}} \boldsymbol{\kappa}^{T}+\Gamma_{\mathrm{gg}}^{-1} \\
\boldsymbol{\lambda}=\boldsymbol{\kappa} \Gamma_{e g} \Gamma_{g g}^{-1}+\Gamma_{\mathrm{gg}}^{-1}
\end{array}\right.
$$

Finally, a MAP estimation for $g(x)$ can be reconstructed from elements of $\mathbf{g}^{M A P}$. This process depends on the selection of mode functions $\varphi_{1}, \ldots, \varphi_{N}$. In this work, local piece-wise linear (hat) functions as shown in Fig. 1a are used as mode functions; thus, $g(x)$ at $x_{l}=\frac{l-1}{N-1}$ can be reconstructed by

$$
g\left(x_{l}\right)=\mathrm{g}_{l}^{M A P}
$$




\section{PROPOSED PARAMETER ESTIMATION ALGORITHM}

Referring to Eq. (3), the second sub-problem is a forward problem which can be solved numerically. To find a solution to this problem, $m(x)$ is written in the modal form of

$$
m(x)=\sum_{k=1}^{N} \mathrm{~b}_{k} \varphi_{k}(x)
$$

where $\varphi_{1}, \ldots, \varphi_{N}$ are mode functions and $b_{1}, \ldots, b_{N}$ are scalar coefficients. Substituting Eq. (16) into Eq. (3) and using Galerkin method results in

$$
-\mathbf{Q b}=\mathbf{f} \Rightarrow \mathbf{b}=-\mathbf{Q}^{-1} \mathbf{f}
$$

where $\mathbf{Q}=\left[\mathrm{Q}_{k l}\right]_{N \times N}$ and $\mathbf{f}=\left[\mathrm{f}_{l}\right]_{N \times 1}$ are given by $\mathrm{Q}_{k l}=\varphi_{k}^{\prime}(0) \varphi_{l}(0)+\int_{0}^{1} \varphi_{k}^{\prime}(x) \varphi_{l}^{\prime}(x) \mathrm{d} x$ and $\mathrm{f}_{l}=\int_{0}^{1} f(x) \varphi_{l}(x) \mathrm{d} x$ and $\mathbf{b}=\left[\mathrm{b}_{l}\right]_{N \times 1}$. Substituting Eq. (23) into (23) results in a solution for $m(x)$. Now, by using the coupling equation given in Eq. (4) and the solutions obtained for $g(x)$ and $m(x)$ in Eqs. (22) and (23) respectively, $\beta(x)$ can be reconstructed by

$$
\beta\left(x_{l}\right)=\frac{1}{\mathrm{~g}_{l}^{M A P}} \sum_{k=1}^{N} \mathrm{~b}_{k} \varphi_{k}\left(x_{l}\right) \quad l=1, \ldots, N
$$

Since $\mathbf{g}_{*}$ represents the best guess for $\mathbf{g}$ at time index $t$, Eq. (20) can be realized by

$$
\mathbf{g}^{(t+1)}=\boldsymbol{\Lambda}^{-1}\left[\boldsymbol{\kappa}\left(\mathbf{r}-\mathbf{e}_{*}\right)+\lambda \mathbf{g}^{(t)}\right]
$$

Combining Eqs. (25) and (26) result in the following parameter estimation algorithm of Euler-Bernoulli beams.

$$
\beta^{(t+1)}\left(x_{l}\right)=\frac{1}{\mathrm{~g}_{l}^{(t+1)}} \sum_{k=1}^{N} \mathrm{~m}_{k} \varphi_{k}\left(x_{l}\right) \quad l=1, \ldots, N
$$

where $b_{1}, \ldots, b_{N}$ and $g_{1}^{(t+1)}, \ldots, g_{N}^{(t+1)}$ are calculated from Eq. (24) and Eq. (26) respectively. The only problem remained is to estimate the statistical parameters used in Eq. (15), which is discussed in the following. It is assumed that the actual beam can be perfectly modeled by using a Finite Element (FE) model with piece-wise linear basis functions and sufficiently high number of equally spaced nodes. A set of numbers from a standard Gaussian distribution is drawn and filtered by using a lowpass filter to produce the nodal values of $g(x)$ as a stochastic process. By using the selected basis functions and nodal values of $g(x)$, Eq. (2) can be solved for the values of the deflection function at the given measurement points $x_{1}, \ldots, x_{M}$. The obtained data is used to form a sample of the measurement vector $\mathbf{r}$. Now, we develop a rough model for the measurement data by considering same nodal values of $g(x)$ but a limited number of equally spaced nodes. Solving this model results in a rough estimate of the measurement vector $\left(\mathbf{r}_{o}\right)$. Some white noise can be added to $\mathbf{r}_{o}$ as measurement noise. Finally, subtracting $\mathbf{r}_{o}$ from $\mathbf{r}$ gives a sample of the approximation error vector $\mathbf{e}$. The above process can be repeated for different independent and identically distributed samples of nodal values of $g(x)$. By using the generated data, $\mathbf{e}_{*}, \mathbf{g}_{*}, \Gamma_{\text {ee }}, \Gamma_{\mathrm{gg}}, \Gamma_{\mathrm{eg}}, \Gamma_{\mathrm{ge}}$ and consequently $\boldsymbol{\kappa}, \boldsymbol{\Lambda}$ and $\boldsymbol{\lambda}$ can be computed.

\section{COMPUTER SIMULATION}

In the computer simulation discussed here, it is assumed that a uniform force $f(x)=1$ is applied to a beam of length 1 and there are 9 equally spaced measurement points at $0.1, \ldots, 0.9$ in the beam. The actual beam is modeled by using piece-wise linear basis functions and 200 equally spaced nodes (considered as a perfect model). A rough model is formed by using 21 equally spaced nodes. Based on the instruction given in the last paragraph of Section $4, \mathbf{e}_{*}, \mathbf{g}_{*}, \Gamma_{\mathrm{ee}}, \Gamma_{\mathrm{gg}}, \Gamma_{\mathrm{eg}}, \Gamma_{\mathrm{ge}}$ and, thereby, $\boldsymbol{\kappa}, \boldsymbol{\Lambda}$ and $\boldsymbol{\lambda}$ are computed by using these the perfect and rough model described above.

Now, we consider a flexural rigidity function $\beta(x)$ as shown by solid blue line in the plot shown in bottom of Fig. 1b. The beam deflection is calculated by using the perfect model and shown in the top plot of Fig $1 b$. The measurement vector is generated by using the values of the deflection function at measurement points (obtained 

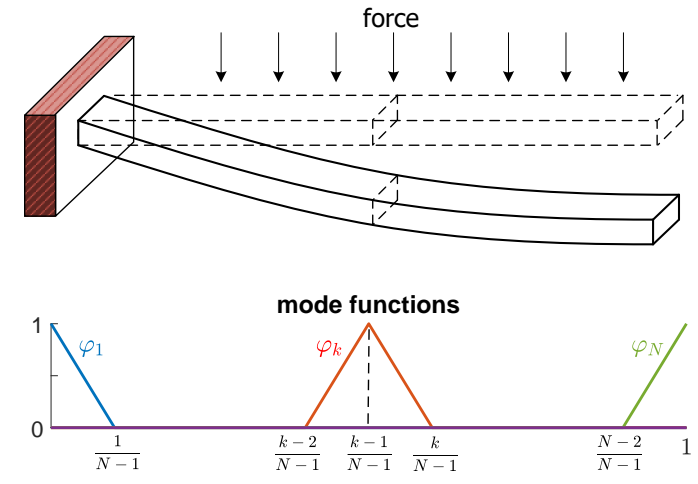

(a) cantilever Euler-Bernoulli beam (top) and piecewise linear basis functions (bottom)
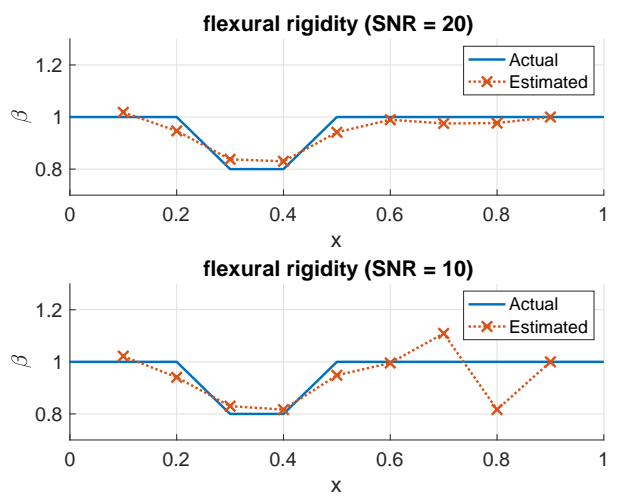

(a) parameter estimation by using the proposed method (top: SNR $=20$ ) and (bottom: SNR = 10)
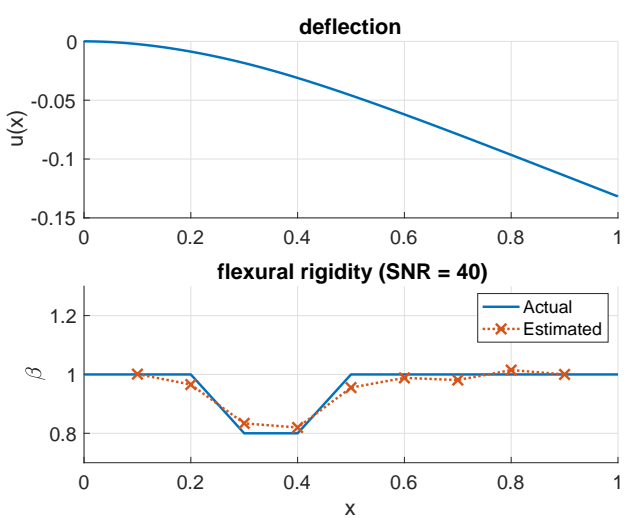

(b) parameter estimation by using the proposed method $(\mathrm{SNR}=50)$.

Figure 1

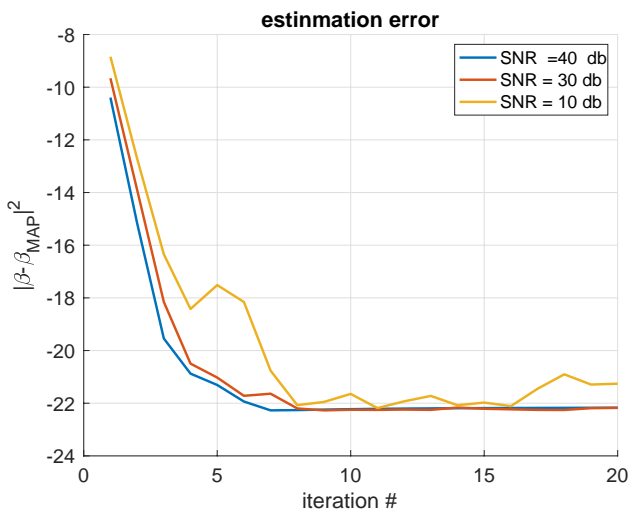

(b) estimation error of the proposed algorithm for different SNR levels

Figure 2

by solving the perfect model) and adding some white noise. In the first experiment, the SNR of the measurement vector is set to $40 \mathrm{db}$. The flexural rigidity is reconstructed by using the algorithm given in Eq. (27). In each iteration, the measurement data is corrupted by using independent and identically distributed noise; however, the SNR is fixed to $40 \mathrm{db}$. After about 20 iteration, the proposed parameter estimation algorithm converges to its final solution. The result is shown by a dashed red line in the bottom plot of Fig. 1b. Also, the estimation error at each iteration is shown in Fig. 2b. As seen, the estimation error becomes -22 db after about 20 iteration.

In the second and third experiments, the SNR is set to $20 \mathrm{db}$ and $10 \mathrm{db}$ respectively. The final solution obtained by using the proposed parameter estimation algorithm for each SNR level is shown in Fig. 2a. As seen, by decreasing the SNR the accuracy of the parameter estimation algorithm is decreased. Also, the convergence behavior of the estimation error for each SNR level is shown in Fig. 2 b.

\section{CONCLUSION}

Unlike force reconstruction, parameter estimation of structural elements such as Euler-Bernoulli beams involves in a nonlinear inverse problem. The nonlinear inverse problem associated with the parameter estimation of Euler-Bernoulli beams can be modeled by using a fourth-order differential equation. By splitting the original problem into two coupled reduced order problems the fourth-order nonlinear inverse problem will be converted 
to a second order linear inverse problem and a second order forward problem. Bayesian Approximation Error approach can efficiently solve the reduced order inverse sub-problem. This approach can consider modeling error unlike traditional regularization approaches. Moreover, this approach does not involve in the adjustment of any regularization parameter. The obtained solution can be reached by using an efficient signal processing algorithm.

\section{REFERENCES}

[1] C. Hansen, "The truncated svd as a method for regularization," BIT Numerical Mathematics, vol. 27, no. 4, pp. 534-553, Dec. 1987. 1

[2] A. N. Tikhonov, "Solution of incorrectly formulated problems and the regularization method," Soviet Mathematics Doklady, vol. 4, pp. 1035-1038, 1963. 1

[3] J. Sanchez and H. Benaroya, "Review of force reconstruction techniques," Journal of Sound and Vibration, vol. 333, no. 14, pp. 2999 - 3018, 2014. 1

[4] E. Jacquelin, A. Bennani, and P. Hamelin, "Force reconstruction: analysis and regularization of a deconvolution problem," Journal of Sound and Vibration, vol. 265, no. 1, pp. 81 - 107, 2003. 1

[5] A. J. Fleming and S. O. R. Moheimani, "Spatial system identification of a simply supported beam and a trapezoidal cantilever plate," IEEE Transactions on Control Systems Technology, vol. 11, no. 5, pp. 726-736, Sept 2003. 1

[6] D. Lesnic, L. Elliott, and D. Ingham, "Analysis of coefficient identification problems associated to the inverse euler-bernoulli beam theory," IMA Journal of Applied Mathematics, vol. 62, no. 2, pp. 101-116, 1999. 1

[7] D. Lesnic, "Determination of the flexural rigidity of a beam from limited boundary measurements," Journal of Applied Mathematics and Computing, vol. 20, no. 1-2, pp. 17-34, 2006. 1

[8] T. T. Marinov and A. S. Vatsala, "Inverse problem for coefficient identification in the euler-bernoulli equation," Computers $\&$ Mathematics with Applications, vol. 56, no. 2, pp. 400 - 410, 2008. 1

[9] T. T. Marinov and R. Marinova, "Inverse problem for coefficient identification in euler-bernoulli equation by linear spline approximation," in Large-Scale Scientific Computing, I. Lirkov, S. Margenov, and J. Waśniewski, Eds. Berlin, Heidelberg: Springer Berlin Heidelberg, 2010, pp. 588-595. 1

[10] J. Kaipio and E. Somersalo, "Statistical inverse problems: Discretization, model reduction and inverse crimes," Journal of Computational and Applied Mathematics, vol. 198, no. 2, pp. 493 - 504, 2007, special Issue: Applied Computational Inverse Problems. 1 